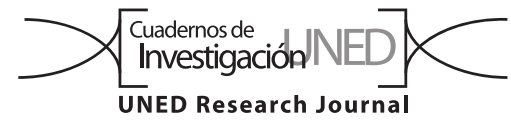

\title{
Riesgo ante el cambio climático de la pesca artesanal y el turismo comunitario en el Golfo de Montijo, Panamá
}

\author{
Antonio Clemente Beyer'1, Alejandro Jiménez Hernández² ${ }^{2}$ \& Vicente Del Cid Mendoza ${ }^{1}$ \\ 1. Fundación MarViva, Panamá; antonio.clemente@marviva.net, vicente.delcid@marviva.net, \\ 2. Consultor independiente; ale.jimenezz@gmail.com
}

Recibido 08-VIII-2018 • Corregido 30-XI-2018 • Aceptado 14-I-2019

\begin{abstract}
Climate change risk of artisanal fishing and community led tourism in the Gulf of Montijo, Panamá. Climate change threatens artisanal fisheries and small-scale marine and coastal tourism as well as the folks who depend on these activities, particularly in developing regions. Urgency lies in adopting measures to ensure these livelihoods and conserve the natural resources on which they depend. MarViva Foundation selected community-based fishing and tourism associations from the Gulf of Montijo on Panama's Pacific coast for climate change adaptation measure implementation support based on their degrees of climate change risk. MarViva estimated degrees of risk of the fishing and tourism sectors by modeling three components that upon interacting generate climate change risk - vulnerability, hazard and exposure. The main result, a community-scale climate change risk index, provided an objective criterion for selecting beneficiary groups and laid the groundwork for designing specific interventions aimed at building resilience and reducing climate change risk drivers relevant to the artisanal fishing and small-scale tourism sectors in the Gulf of Montijo.
\end{abstract}

Key words: adaptation, risk, vulnerability, hazard, exposure, Eastern Tropical Pacific, climate change, Panama.
RESUMEN: El cambio climático amenaza a las actividades de pesquerías artesanales y de turismo marino y costero a pequeña escala, poniendo en riesgo a las personas que dependen de estas actividades económicas, particularmente en regiones en vías de desarrollo. Por lo tanto, urge adoptar medidas para asegurar estos medios de vida y conservar los recursos naturales de los cuales dependen. Fundación MarViva eligió asociaciones de base comunitaria dedicadas a la pesca y al turismo en el Golfo de Montijo, Pacífico de Panamá en función de sus niveles de riesgo ante el cambio climático, para apoyar con la implementación de medidas de adaptación. A fin de cuantificar el riesgo, se modeló este concepto para los sectores de pesca y turismo a través de la valoración de tres componentes que al interactuar, generan el riesgo ante el cambio climático - vulnerabilidad, peligro y exposición. El resultado, un índice de riesgo ante el cambio climático a escala de comunidad, proporcionó un criterio objetivo con respecto a la selección de grupos beneficiarios y cimentó las bases del diseño de intervenciones específicas dirigidas a aumentar la resiliencia y a reducir las causas subyacentes del riesgo.

Palabras claves: adaptación, riesgo, vulnerabilidad, peligro, exposición, Pacífico Tropical Oriental, cambio climático, Panamá.
El cambio climático amenaza a las pesquerías artesanales y al turismo a pequeña escala, así como a las personas que dependen de estas actividades económicas. Este se suma a las presiones concurrentes como la sobrepesca, la degradación de hábitats, la contaminación de aguas y costas y la introducción de especies invasoras (Brander, 2010; Whitty, 2014). Manifestaciones del cambio climático como el ascenso del nivel del mar, el aumento de intensidad y frecuencia de eventos meteorológicos extremos y una variación compleja en la dinámica de condiciones oceanográficos (ej. salinidad, $\mathrm{pH}$, temperatura, circulación), están impactando a los ecosistemas marinos, a las poblaciones de peces e invertebrados, a la actividad de pescadores y prestadores de servicios turísticos y a la infraestructura física de apoyo con que cuentan las comunidades costeras (IPCC, 2014; Whitty, 2014).

En el 2014, Fundación MarViva desarrolló la investigación "Cartografiando los hábitats marino-costeros críticos vulnerables a los impactos del cambio climático en el Golfo de Chiriquí", la cual ubicó zonas vulnerables al ascenso del nivel del mar en el Golfo de Montijo (GdM), un área de importante actividad pesquera y turística en el litoral Pacífico de Panamá. De allí surgió el proyecto "Adaptando la pesca y el turismo del Golfo de Montijo al cambio climático", que fue financiado por el Banco Interamericano de Desarrollo, orientado a brindar estrategias de adaptación al cambio climático en una 
población carente de alternativas económicas y en desconocimiento del tema.

El desarrollar e implementar medidas de adaptación con recursos finitos para una región con más de 20 asociaciones de base comunitaria dedicadas a la pesca y/o el turismo (Ministerio de Ambiente, 2014) implica retos. Un apoyo fundamental lo daría una herramienta que permita priorizar a qué grupos amparar, en función de su nivel de riesgo ante el cambio climático. De allí surge la presente investigación aplicada.

En el contexto de cambio climático, peligro se define como el acontecimiento potencial de un suceso o tendencia física relacionada con el clima o los impactos de este, que pueden causar pérdidas y daños; por ejemplo, el aumento de temperatura media o mayor frecuencia y/o intensidad de eventos extremos asociados a la variabilidad climática. Es así que "peligro" es equivalente al concepto de "amenaza". Por su lado, exposición es la presencia de algo de valor en lugares y entornos que podrían verse afectados por los peligros o amenazas existentes en el área. Vulnerabilidad, por su parte, es la propensión o predisposición a ser afectado negativamente, mientras que riesgo se refiere al potencial de consecuencias debido a la exposición ante un peligro inminente con un desenlace incierto (IPCC, 2014). El riesgo ante el cambio climático se deriva de la interacción de los peligros, con la vulnerabilidad y exposición de los sistemas humanos y naturales (IPCC, 2014).

El presente estudio tiene como objetivo modelar y representar la distribución espacial del riesgo climático de las actividades de pesca y de turismo en el GdM a una escala de comunidad productiva, basándose en la caracterización y valoración de sus componentes: vulnerabilidad, peligros y exposición, mediante el desarrollo de un índice comprehensivo de riesgo ante el cambio climático.

\section{METODOLOGÍA}

Para lograr el objetivo se han utilizado indicadores de vulnerabilidad (factores que inciden en la susceptibilidad, sensibilidad y capacidad adaptativa), información sobre peligros (¿cuáles son los peligros o amenazas relacionados al cambio del clima que afectan a la pesca y al turismo costero y dónde se presentan?) e información sobre las áreas donde se pesca y se realiza el turismo (para definir la exposición). Se recurrió a fuentes secundarias, reuniones con expertos, recorridos por el litoral del Golfo y consulta a miembros de asociaciones comunitarias dedicadas a la pesca artesanal y/o la prestación de servicios de turismo marino y costero en el Golf de Montijo a fin de reunir estos insumos. Los datos sobre las áreas de pesca y turismo así como los indicadores de vulnerabilidad debían ser diferentes para cada comunidad o, por lo menos, estar a nivel de corregimiento, para generar resultados que reflejan la variación de los niveles de riesgo entre las comunidades.

Se convocó a microempresarios turísticos claves y a las principales asociaciones comunitarias dedicadas a la pesca a dos talleres, a fin de levantar datos de percepción sobre vulnerabilidad, peligros y exposición. Los participantes provinieron de las 20 comunidades que se eligieron como sujeto de estudio. La dinámica facilitó una discusión guiada a través del cual se definieron los seis peligros relacionados al clima más relevantes para estas actividades productivas, incluyendo condiciones de variabilidad climática conocidos como 'mal tiempo', cuya frecuencia y/o intensidad se percibe que tiende a aumentar debido al cambio climático. Luego, mediante cartografía participativa se mapeó la distribución geográfica de estos peligros principales, con excepción del ascenso del nivel del mar (ANM). Los mapas comunitarios de peligro se digitalizaron a formato raster, asignándole un valor de 5 a cada uno donde el peligro se manifiesta con máxima intensidad. Los valores disminuyen a lo largo de la superficie, al reducirse la intensidad del peligro en dado lugar, según indicado por los participantes. Se utilizó este método basado en la percepción de los usuarios sobre los peligros del cambio climático en la actualidad, debido a que no existen proyecciones de cambio climático basadas en los escenarios del IPCC a una escala lo suficiente fina y que contemplen los peligros más relevantes para las actividades productivas en consideración. Los participantes mapearon las zonas específicas usadas para la pesca y/o el turismo por cada comunidad, generando un insumo que posteriormente al compararse con la distribución espacial de los peligros, defina el concepto de exposición. También se valoró la sensibilidad de las actividades de pesca y turismo a los peligros principales a fin de aportar al análisis de vulnerabilidad, esto mediante la asignación de valores entre 1 (sensibilidad mínima) y 5 (sensibilidad máxima) por parte de los participantes para la pesca e independientemente para el turismo, con respecto a cada peligro y cada comunidad. Se calculó un valor promedio de sensibilidad por comunidad, ponderando mayor peso a los valores correspondientes a la sensibilidad de la actividad dominante en la comunidad (pesca o turismo).

El peligro producido por el ANM se mapeó considerando elevación ( $<5 \mathrm{~m}$ sobre el nivel del mar), subsidencia y erosión costera. Como insumo se empleó un modelo digital del terreno WorldDEM DTM ${ }^{\text {TM }}$ (resolución horizontal de aproximadamente $12 \mathrm{~m}$ y exactitud vertical relativa de $<5 \mathrm{~m}$ ), información de zonas padeciendo de 
hundimiento concedida por el Instituto de Geociencias de la Universidad de Panamá (A. Tapia, comm. pers., 28 de octubre, 2016) y un mapa de erosión costera proveniente de observación primaria. A la valoración espacial resultante en formato raster se le asignó un valor máximo de 25. Con el fin de contar con un solo mapa que resumiera la distribución de peligros y fuera comparable con los mapas de uso (áreas donde se pesca y realiza turismo), los cinco mapas de peligro producidos junto a los actores comunitarios y el mapa de peligro por el ANM se combinaron en el Sistema de Información Geográfica (SIG). Esta sumatoria comprende la valoración espacial que representa la intensidad de los peligros principales mediante un gradiente de color (valor más cercano al máximo = rojo más intenso). El valor máximo de la sumatoria de los 5 peligros marinos $(5+5+5+5+5=25)$ equivale al valor máximo del único peligro en tierra firme considerado, resultando en un rango parejo para ambos medios a pesar de considerar mayor cantidad de peligros sobre el medio marino.

A fin de contar con valoraciones de vulnerabilidad disgregados por comunidad, se desarrolló un índice tabulado mediante una metodología nueva, producto del promedio de tres variables conexas a la susceptibilidad, sensibilidad y capacidad adaptativa, una proveniente de los talleres (sensibilidad a peligros) y dos de fuentes externas (indicador económico, indicador de nivel de educación y condiciones de vivienda). Para ello se procesaron los datos según su formato, generando un valor por comunidad para cada insumo (por ejemplo, el indicador de nivel de educación y condiciones de vivienda consolida varios datos del censo). Los valores de cada insumo que corresponden a cada una de las 20 comunidades se normalizaron a un rango entre cero a uno. Finalmente se calculó el promedio de los valores normalizados de los tres insumos $[(\mathrm{v} 1+\mathrm{v} 2+\mathrm{v} 3) / 3]$ y se normalizó el resultado al mismo rango.

La exposición de cada comunidad se valoró en función de la intensidad de los peligros que pueden afectar sus zonas de pesca y/o turismo, así como por el grado de peligro por el ANM de la ubicación de la comunidad. Para ello se sobrepusieron los mapas de uso (zonas de pesca y/o turismo) y sitios de las comunidades con el mapa de intensidad de peligros. Luego, mediante interpretación visual, se le asignó un coeficiente de exposición del 1 (mínimo) al 5 (máximo) a cada comunidad. Es decir, a cada

Ecuación convierte un valor dentro de un rango determinado a un valor proporcional en el rango entre cero y uno, procedimiento definido como normalización.

$\mathrm{x}=$ valor

max = valor máximo del rango

$\min =$ valor mínimo del rango 

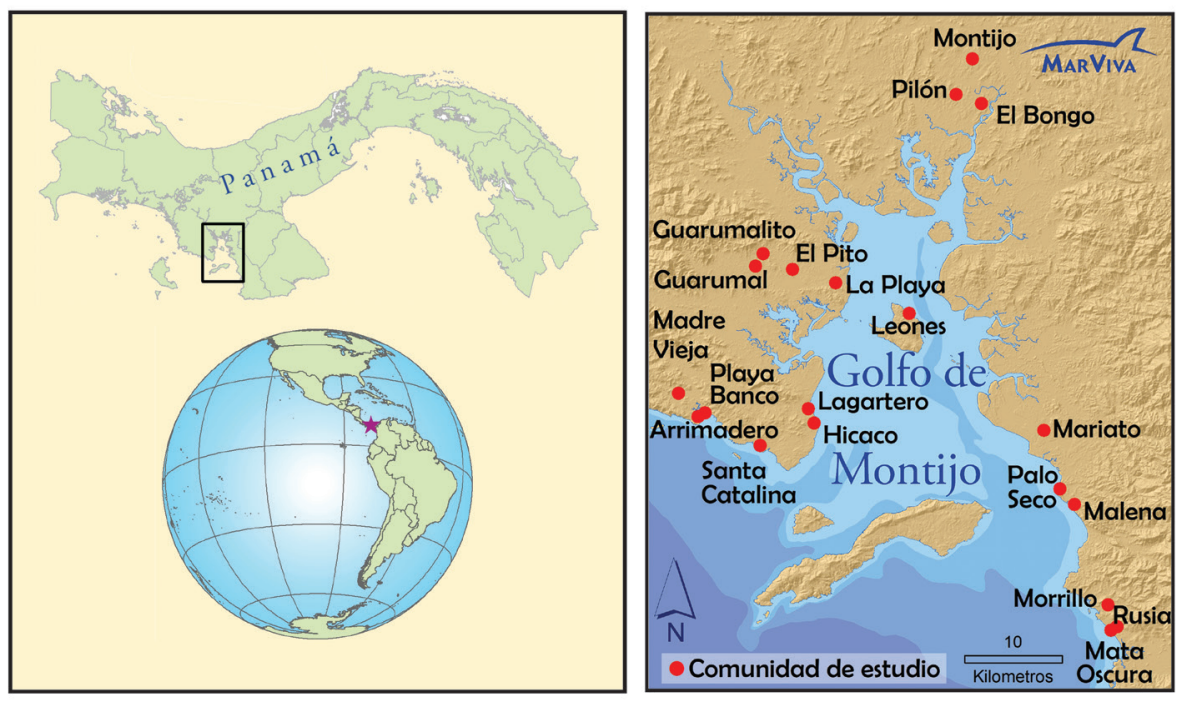

Fig. 1. Localización del Golfo de Montijo y de las comunidades de estudio.

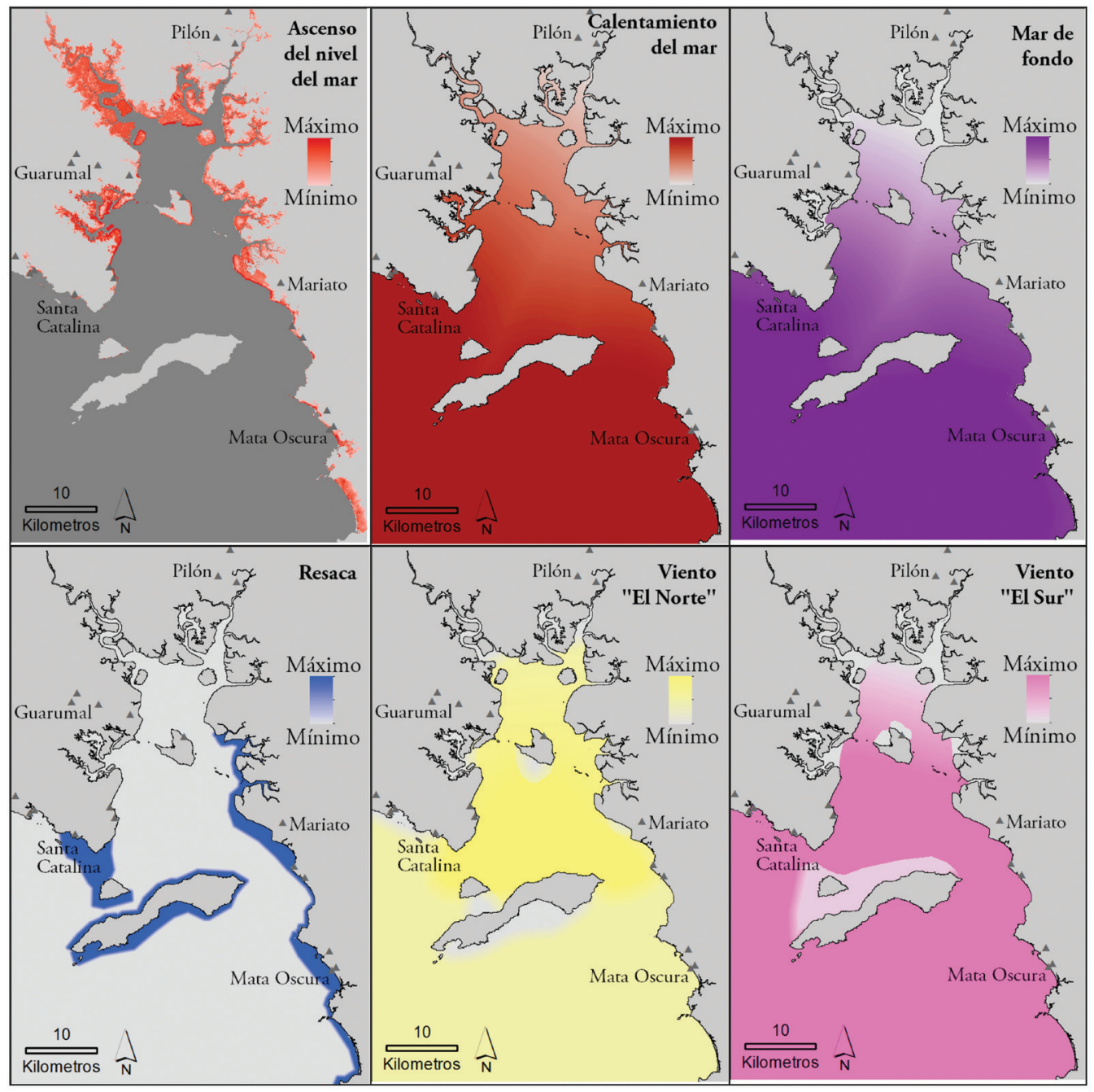

Fig. 2. Peligros de variabilidad y cambio climático más relevantes para las actividades de pesca y turismo. 


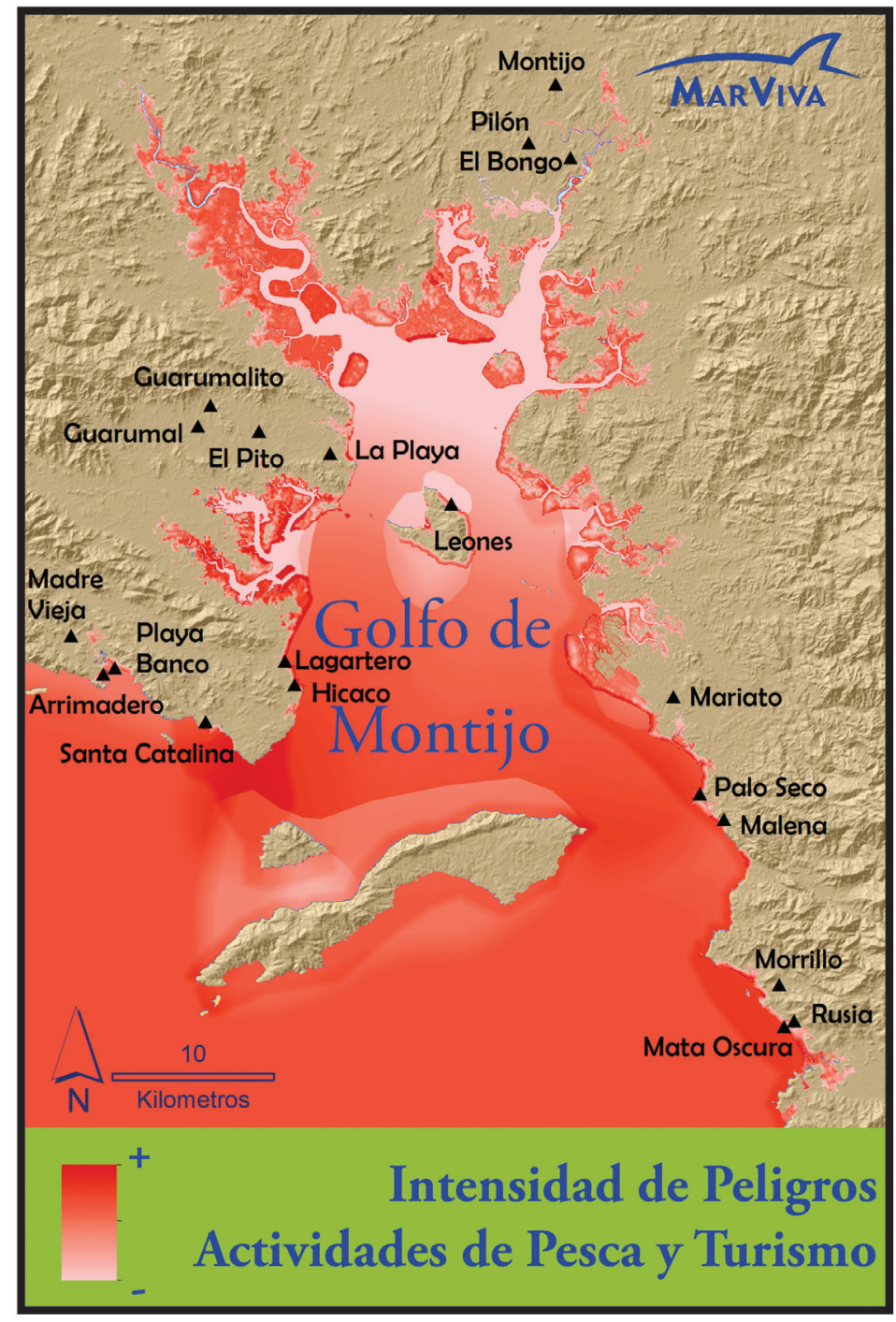

Fig. 3. Distribución e intensidad de peligros.

Hicaco, presenta los valores más elevados de intensidad de peligros.

\section{Exposición de comunidades a peligros principales}

La exposición de la pesca y turismo se precisa para cada comunidad a través de un coeficiente de valor entero (1-5), el cual se definió visualmente con base en los mapas comunitarios de zonas de uso sobrepuestos al mapa compuesto de peligros. Cinco comunidades coincidieron en el coeficiente mayor de exposición a peligros (cinco), once coincidieron en el valor siguiente (cuatro), tres en el valor correspondiente a exposición media (tres) y una en el valor moderado (dos).

\section{Índice de vulnerabilidad}

La vulnerabilidad de la pesca y el turismo se califica por medio de un índice que sintetiza tres variables y permite comparar grados de vulnerabilidad entre comunidades. El valor uno $(1,00)$ del índice de vulnerabilidad corresponde a la comunidad calificada con el mayor grado de vulnerabilidad. El valor uno $(1,00)$ de un insumo (tres primeras columnas del cuadro 1) señala la comunidad con el mayor grado de pobreza, de sensibilidad a peligros, o de analfabetismo, baja escolaridad y viviendas carecientes de servicios básicos.

Variables del índice de vulnerabilidad (Cuadro 1):

1. Índice económico basado en proporción de población en situación de pobreza extrema (egresos menores a \$534/año) (CIESIN, 2007). 
CUADRO 1

Valoraciones del índice de vulnerabilidad de la pesca y el turismo e insumos

\begin{tabular}{|c|c|c|c|c|}
\hline Comunidad & Índice económico & Índice sensibilidad & Índice educación vivienda & Índice vulnerabilidad \\
\hline Morrillo & 1,00 & 1,00 & 0,80 & 1,00 \\
\hline El Banco & 0,92 & 0,67 & 1,00 & 0,90 \\
\hline Arrimadero & 0,92 & 0,67 & 0,91 & 0,86 \\
\hline Leones & 0,67 & 0,94 & 0,73 & 0,78 \\
\hline Mata Oscura & 1,00 & 1,00 & 0,34 & 0,78 \\
\hline Lagartero & 0,92 & 1,00 & 0,36 & 0,76 \\
\hline Hicaco & 0,92 & 0,81 & 0,38 & 0,67 \\
\hline Malena & 0,77 & 1,00 & 0,19 & 0,61 \\
\hline Santa Catalina & 0,92 & 0,83 & 0,18 & 0,59 \\
\hline Rusia & 1,00 & 0,70 & 0,21 & 0,58 \\
\hline Madre Vieja & 0,92 & 0,67 & 0,32 & 0,58 \\
\hline Mariato & 0,77 & 0,80 & 0,28 & 0,56 \\
\hline Palo Seco & 0,77 & 0,50 & 0,33 & 0,44 \\
\hline Guarumalito & 0,66 & 0,33 & 0,44 & 0,35 \\
\hline La Playa & 0,66 & 0,33 & 0,33 & 0,30 \\
\hline Guarumal & 0,66 & 0,33 & 0,23 & 0,25 \\
\hline El Pito & 0,66 & 0,00 & 0,38 & 0,17 \\
\hline El Bongo & 0,24 & 0,60 & 0,11 & 0,13 \\
\hline Montijo Centro & 0,24 & 0,60 & 0,00 & 0,08 \\
\hline Pilón & 0,00 & 0,60 & 0,07 & 0,00 \\
\hline
\end{tabular}

CUADRO 2

Valoraciones del índice de riesgo ante el cambio climático e insumos

\begin{tabular}{|c|c|c|c|}
\hline Comunidad & Índice vulnerabilidad & Coeficiente exposición & Índice riesgo \\
\hline Morrillo & 1,00 & 5 & 1,00 \\
\hline Mata Oscura & 0,78 & 5 & 0,95 \\
\hline Malena & 0,61 & 5 & 0,92 \\
\hline Rusia & 0,58 & 5 & 0,91 \\
\hline Palo Seco & 0,44 & 5 & 0,88 \\
\hline El Banco & 0,90 & 4 & 0,67 \\
\hline Arrimadero & 0,86 & 4 & 0,67 \\
\hline Leones & 0,78 & 4 & 0,65 \\
\hline Lagartero & 0,76 & 4 & 0,64 \\
\hline Hicaco & 0,67 & 4 & 0,63 \\
\hline Santa Catalina & 0,59 & 4 & 0,61 \\
\hline Mariato & 0,56 & 4 & 0,60 \\
\hline Guarumalito & 0,35 & 4 & 0,56 \\
\hline La Playa & 0,30 & 4 & 0,55 \\
\hline Guarumal & 0,25 & 4 & 0,54 \\
\hline El Pito & 0,17 & 4 & 0,52 \\
\hline El Bongo & 0,13 & 3 & 0,21 \\
\hline Montijo Centro & 0,08 & 3 & 0,20 \\
\hline Pilón & 0,00 & 3 & 0,18 \\
\hline Madre Vieja & 0,58 & 2 & 0,00 \\
\hline
\end{tabular}


2. Índice de sensibilidad de la pesca y/o turismo ante los principales peligros, basado en percepción comunitaria (fuente primaria).

3. Índice de educación y vivienda basado en métricas del Censo 2010 (población analfabeta / mayor de diez años con menos de tercer grado escolar; viviendas sin agua / servicio sanitario / electricidad) (INEC, 2011)

\section{Índice de riesgo}

El índice de riesgo califica el riesgo ante el cambio climático de la pesca y el turismo de comunidades del GdM mediante una valoración que permite comparar los niveles entre comunidades. El valor uno $(1,00)$ corresponde a la comunidad calificada con el mayor grado de riesgo climático. Este índice modela la influencia de los tres componentes del riesgo: la vulnerabilidad, exposición y peligro, mediante la combinación, a través de un promedio ponderado, del índice de vulnerabilidad y el coeficiente de exposición a peligros (Cuadro 2 y Figura 4).

\section{ANÁLISIS DE RESULTADOS}

Este estudio evalúa el riesgo ante el cambio climático de forma integral mediante la modelación de sus componentes subyacentes, vulnerabilidad, peligro y exposición, y genera una herramienta de apoyo para la toma de decisión, el índice de riesgo ante el cambio climático de la pesca y el turismo.

Las valoraciones tanto del índice de vulnerabilidad como del índice de riesgo no corresponden a niveles absolutos, sino a niveles comparativos. Por lo tanto, el valor cero se debe interpretar como "menos que las demás comunidades de estudio", y no "sin vulnerabilidad" o "sin riesgo". Centrar el estudio en dos actividades que

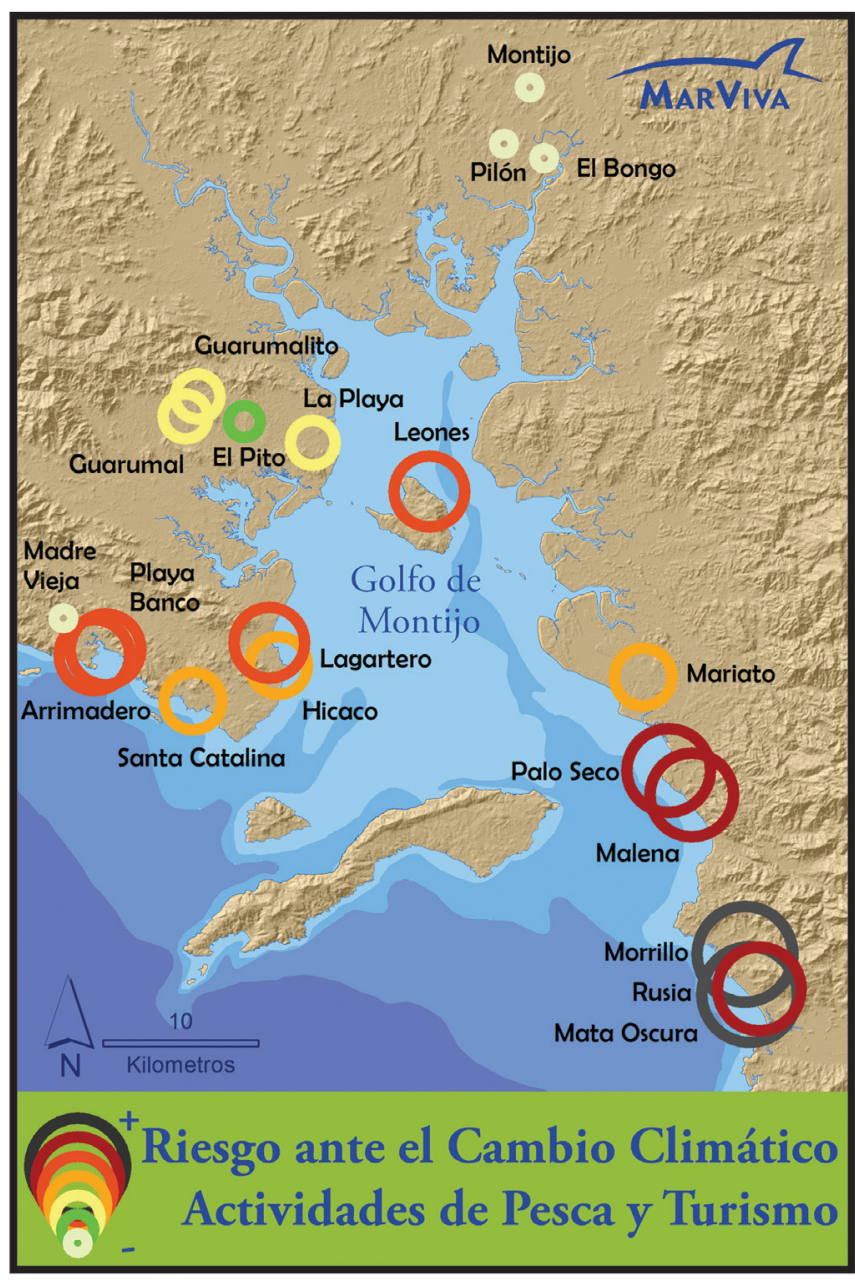

Fig. 4. Riesgo ante el cambio climático de las actividades de pesca y turismo a escala de comunidad productiva, según el índice de riesgo (Cuadro 2). 
dependen de la navegación de embarcaciones pequeñas permitió ampliar su alcance sin reducir la relevancia de los resultados, dado que los peligros principales coinciden y los métodos para la valoración de la sensibilidad y exposición a peligros consideran ambos sectores por separado. Hubo una excepción, el calentamiento marino, pues este peligro interfiere con la pesca al influenciar el comportamiento de los peces, más no el turismo.

Con respecto al trabajo participativo, levantar datos de percepción por medio de talleres comunitarios fue práctico, pero conlleva una debilidad en cuanto a la homogeneidad de los datos ya que el conocimiento de un pescador o botero turístico puede no ser representativo de su comunidad.

Una validación del índice de riesgo que podría llevarse a cabo mediante monitoreo de afectaciones generaría mayor confianza en el resultado y permitiría la calibración de la ponderación empleada en la síntesis de factores mediante promedios. Dado que algunos grupos involucrados en el presente estudio se encuentran implementando medidas de adaptación en marco del mismo proyecto, se espera una reducción de las afectaciones futuras, hecho que interferiría con la metodología de validación propuesta. No obstante, se recomienda considerar algún componente de validación en cualquier estudio que construya sobre esta metodología.

\section{Índice de vulnerabilidad}

Los insumos del índice de vulnerabilidad abarcan tres factores subyacentes asociados a la vulnerabilidad: sensibilidad, capacidad adaptativa y susceptibilidad (IPCC, 2014). Haber incluido dos insumos socioeconómicos generales y uno enfocado en las actividades productivas de interés permitió que el índice de vulnerabilidad asimile ambos ejes pertinentes, la comunidad como la base y los medios productivos como actividades de enfoque. Incluir un abanico más amplio de insumos fortalecería los resultados; lamentablemente fueron escasas las fuentes cuyo nivel de detalle llegase a la escala de la comunidad o del corregimiento.

La normalización de los valores de los insumos al rango de cero a uno se realizó a fin de evitar distorsionar los resultados del promedio.

\section{Peligros principales}

Si bien algunos de los peligros definidos como principales se tratan de condiciones de mal tiempo y no cambios en la media del clima, y el tiempo no se debe confundir con el clima, existe una tendencia asociada al cambio climático hacia el aumento en la intensidad y la frecuencia de eventos de mal tiempo, (Sousounis \& Little, 2017). El hecho, justamente, que varios de los peligros climáticos que quedaron entre los principales para la pesca y el turismo costero son más bien condiciones de mal tiempo resalta la relevancia de la Reducción de Riesgo de Desastres como medida de adaptación al cambio climático. Llama la atención que los dos peligros del cambio climático más comúnmente considerados, el aumento de temperatura superficial del aire y cambios de patrones de precipitación, no hayan quedado priorizados para los sectores de la pesca y el turismo marino y costero.

\section{Coeficiente de exposición a peligros}

El carácter escalonado y repetido de los coeficientes de exposición evidencia una deficiencia del método que los definió. La precisión y exactitud de la valoración aumentaría si se reemplazara la interpretación visual por un análisis espacial matemático en SIG que generase una serie más variada de coeficientes en función de la intensidad de peligros en las zonas de uso.

Una vez adelantado el estudio se dio a conocer que desde la comunidad de "Madre Vieja" no se realiza la pesca o el turismo directamente; algunas personas dedicadas al turismo en la comunidad de "Arrimadero" se albergan en "Madre Vieja". Estando menos vinculado a las actividades productivas de enfoque, se decidió asignarle un coeficiente moderado de exposición (2 de 5).

\section{Índice de riesgo ante el cambio climático}

De acuerdo con una ponderación dominante del factor exposición a peligros, se aprecia que la sucesión de los valores del índice de riesgo coincide con la sucesión de los valores de exposición. Al contar con las mayores puntuaciones en cuanto a vulnerabilidad y exposición a peligros, la comunidad de "Morrillo" queda en el primer puesto del índice de riesgo. Con zonas de uso similares y también altamente expuestas, a "Morrillo" le siguen en orden decreciente, "Mata Oscura","Malena", "Rusia" y"Palo Seco". Al coincidir con un coeficiente de exposición de cuatro, la sucesión de las once comunidades siguientes va en orden del índice de vulnerabilidad. Contando con el coeficiente de exposición menor, Madre Vieja queda con el valor menor del índice de riesgo. Siendo un caso anómalo, se pudo haber eliminado Madre Vieja, permitiendo que Pilón ocupase el valor mínimo del índice y que los valores de las demás comunidades se distribuyan más ampliamente dentro del rango de cero a uno. 


\section{CONCLUSIÓN}

Los resultados representan una aproximación hacia una caracterización de la distribución espacial del riesgo ante el cambio climático de grupos comunitarios dedicados a la pesca artesanal y al turismo marino y costero en el GdM y proporcionan un respaldo objetivo con respecto a la selección de los grupos beneficiados por el proyecto "Adaptando la pesca y el turismo del Golfo de Montijo al cambio climático". Se consideran acertados, en el sentido que se alinean a la percepción del equipo de MarViva, cuyo criterio se basa en experiencia amplia apoyando asociaciones de pesca y turismo del GdM.

La contribución del índice resulta de su carácter comprehensivo el cual aporta tanto una calificación de riesgo integral como perspicacia respecto al abanico de mecanismos subyacentes. Justamente, el conocimiento adquirido sobre las bases de la vulnerabilidad, el carácter de los peligros y de la exposición y el juego entre éstos, sobrepasa la etapa del proyecto descrita en el presente estudio y aporta a su próximo paso, el diseño de intervenciones específicas de adaptación dirigidas a reducir algunas de las causas del riesgo en cada una de las comunidades beneficiadas. La metodología desarrollada se recomienda como una herramienta para la caracterización y comparación del riesgo ante el cambio climático de grupos dedicados a sectores productivos específicos, así como cualquier aplicación que se aproveche de una cuantificación integral del riesgo ante el cambio climático y de sus componentes.

\section{REFERENCIAS}

Bell, J., M. Batty, A. Ganachaud, P. Gehrke, A. Hobday, O. HoeghGuldberg, J. Johnson, R. Le Borgne, P. Lehodey, J. Lough, T. Pickering, M. Pratchett, M. Sheaves y M. Waycott, 2009. Preliminary assessment of the effect of climate change on fisheries and aquaculture in the Pacific. Secretariat of the Pacific Community, Noumea, New Caledonia, 15 pp. Recuperado de: http://www.spc.int/sppu/images/stories/preliminary\%20assessment.pdf

Brander, K. (2010). Impacts of climate change on fisheries. Journal of Marine Systems, 79(3-4), 389-402. DOI:10.1016/j.jmarsys.2008.12.015

CIESIN (Center for International Earth Science Information Network - Columbia University). (2007). Poverty Mapping Project: Small Area Estimates of Poverty and Inequality (v1) [Archivo de datos formato tabulado]. Palisades, NY: NASA Socioeconomic Data and Applications Center (SEDAC). DOI:10.7927/h49p2zkm

IPCC (Grupo Intergubernamental de Expertos sobre el Cambio Climático). (2014). Cambio climático 2014: Impactos, adaptación y vulnerabilidad - Resumen para responsables de políticas (Contribución del Grupo de trabajo II al Quinto Informe de Evaluación del Grupo Intergubernamental de Expertos sobre el Cambio Climático). Ginebra, Suiza: Organización Meteorológica Mundial. Recuperado de http://ipcc.ch/pdf/assessment-report/ar5/wg2/ ar5_wgll_spm_es.pdf

INEC (Instituto Nacional de Estadística y Censo). (2011). XI Censo de Población y VII de Vivienda [Archivo de datos formato SIG]. Ciudad de Panamá: Contraloría General de la República de Panamá. Recuperado de http:// datos-geored.opendata.arcgis.com/datasets/0b32d699058d4e83a702e3bd0dc71c4f_0.zip

Ministerio de Ambiente (2014). Plan de Manejo Área de Recursos Manejados Humedal Golfo de Montijo [documento técnico inédito]. Ciudad de Panamá: Fundación MarViva, Conservación Internacional, Fundación Natura.

Sousounis, P., \& Little, C. (2017). Climate Change Impacts on Extreme Weather [documento técnico White paper]. Boston, MA: Air Worldwide Corporation. Recuperado de http://w3.air-worldwide.com/Climate-ChangeImpacts-on-Extreme-Weather?utm_campaign=inline

Whitty, T. S. (2014). Climate Change \& Small-scale Fisheries [infografía]. Recuperado de http://www.oceanscientists.org/ images/14_1114_SAFRN_ClimateChange_COP20.pdf 